\title{
Myeloid RelA regulates pulmonary host defense networks
}

\author{
C. Hess*, C. Herr*, C. Beisswenger*, T. Zakharkina*, R.M. Schmid ${ }^{\#}$ and R. Bals*
}

ABSTRACT: The pulmonary innate immune system in the respiratory tract eliminates inhaled pathogens. Several cell types contribute to host defense within a complex network. The aim of this study was to evaluate the role of macrophages during pneumonia and in the regulation of the epithelial response to microorganisms.

We performed lung infection models in mice lacking myeloid RelA/p65. To study the mechanistic relationships between individual cell types, we applied co-culture models composed of airway epithelial cells (AECs) and macrophages.

Mice lacking myeloid RelA/p65 showed significantly decreased bacterial clearance, cytokine expression and neutrophil influx. In addition, the induction of epithelial keratinocyte chemoattractant expression was blunted in these animals. In vitro, AECs were largely insensitive to ligands of Toll-like receptor (TLR)2 or TLR5. Exposure to secretory products of macrophages results in an increased release of pro-inflammatory cytokines and augmented antimicrobial activity. This was associated with increased expression of TLR genes and surface expression of the proteins. Experiments with blocking antibodies showed that the effect of macrophages depends on secreted mediators, including tumour necrosis factor- $\alpha$.

In conclusion, the present data show that myeloid RelA is critical for pulmonary host defense. One important mechanism is that macrophages induce the sensitivity of AEC's to microbial patterns.

KEYWORDS: Antimicrobial peptide, asthma, chronic obstructive pulmonary disease, innate immunity, toll-like receptor

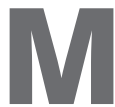
icroorganisms are a potential threat to the integrity of the body and the innate immune system of mucosal surfaces has evolved largely to protect inner and outer body surfaces. The lung is continuously exposed to microorganisms; however, the pulmonary innate immune system is responsible for maintaining a high level of sterility [1]. A breach of pulmonary immune mechanisms results in the development of invasive infection. Pneumonia is a frequent cause of pulmonary morbidity and is associated with significant lethality.

The pulmonary innate immune system is comprised of multiple components, including a number of cell types that fulfil different tasks in host defense [2]. The requirements for the regulation of pulmonary innate immunity are different from other surfaces of the body. During exposure to commensal microorganisms a continuous level of protection must be maintained. In contrast, the presence of organisms with pathogenic or invasive potential can induce a local inflammatory and host defense reaction. In the case of invasive infection, the local defense system provides a first line of protection and activates underlying defense mechanisms, such as influx of phagocytes and stimulation of the adaptive immune system.

Macrophages are professional host defense cells, which are involved in the recognition of and response to microorganisms [3]. In the lung, alveolar macrophages encompass the macrophage population. Airway epithelial cells (AECs) are structural cells and constitute a barrier that keeps the environment physically separated from the internal milieu. In addition, AECs actively contribute to innate immunity. AECs express a number of pattern recognition receptors, including toll-like receptors (TLR) [46] and nucleotide-binding oligomerisation domain proteins [7]. AECs respond to bacteria of different species by activation of inflammatory signalling pathways and the release of proinflammatory mediators and antimicrobial compounds [8]. Several studies have addressed the issue of to what extent different cell types contribute to host defense mechanisms against infection and the issue of development of an
AFFILIATIONS

${ }^{*}$ Dept of Internal Medicine, Division for Pulmonary Diseases, PhilippsUniversität Marburg, Marburg, and \#Dept of Internal Medicine II, Technical University of Munich, Munich, Germany.

CORRESPONDENCE

R. Bals

Dept of Internal Medicine, Division for Pulmonary Diseases Philipps-University Marburg Baldingerstrasse 1 35043 Marburg Germany

E-mail: bals@staff.uni-marburg.de

Received:

Dec 282008

Accepted after revision:

July 282009

First published online: Aug 132009 
inflammatory innate immune reaction using chimeric [9-13] or transgenic [14-16] approaches. These data show that both epithelial/structural cells and professional defense cells have a role in protecting the lung from infection. It is also evident that different cell types interact in different complex ways. Macrophages have been implicated in the regulation of epithelial cell function. Bronchoalveolar lavage fluid (BALF) from pneumonia patients induced the activation of epithelial cells [17]. Conditioned media from activated macrophages has been shown to activate nuclear factor (NF)- $\kappa \mathrm{B}$ in airway epithelial cell lines dependent on tumour necrosis factor (TNF)- $\alpha$ and interleukin (IL)-1 $\beta[18,19]$.

The hypothesis of the present study is that macrophages and epithelial cells are involved in pulmonary host defense. While macrophages are sensitive sentinel cells of immunity, AECs are less sensitive to microbial stimulation and represent a physical barrier. Optimal stimulation of AECs requires a second signal in addition to the presence of the microbial compound, a warning signal that causes the local innate host defense system to switch from continuous noninflammatory protection towards an inflammatory activation of host defense. The aim of the study was to characterise the role of the myeloid NF- $\kappa B$ RelA subunit in host defense cells and to test whether the interaction of lung macrophages and AECs is necessary and sufficient for the induction of an epithelial host defense reaction.

\section{MATERIALS AND METHODS}

\section{Cell culture, media and reagents}

Primary AECs (pAECs) were isolated from large airways resected during surgery and cultivated as submersed or airliquid interface (ALI) cultures as described previously [20]. The protocol was approved by the ethics committee of the University of Munich (Munich, Germany), and informed consent was obtained from the patients. The human epithelial lung cancer cell line A549 and human monocytic cell line U937 were maintained in RPMI 1640 (Invitrogen, Karlsruhe, Germany) supplemented with $10 \%$ fetal calf serum (FCS) (Invitrogen), $100 \mathrm{U} \cdot \mathrm{mL}^{-1}$ penicillin and $0.1 \mathrm{mg} \cdot \mathrm{mL}^{-1}$ streptomycin (Invitrogen). For monocytic differentiation, U937 cells were cultured in the presence of $10 \mathrm{nM}$ phorbol-12myristat13acetat (Sigma Aldrich, Munich). Differentiation of cells was confirmed by the expression of CD11b and CD14 analysed by flow cytometry. Primary macrophages were prepared from peripheral blood mononuclear cells by magnetic cell sorting with anti-CD14 MicroBeads (Miltenyi Biotec, Bergisch Gladbach, Germany). Monocytes adherent to plastic dishes were cultured in RPMI 1640 supplemented with $50 \mathrm{ng} \cdot \mathrm{mL}^{-1}$ macrophage colony stimulating factor (Strathmann Biotec, Hamburg, Germany) for 7 days to allow differentiation to macrophages.

\section{Stimulation protocols}

Supernatants from U937- and monocyte-derived macrophages were prepared by incubation of the cells with or without $100 \mathrm{ng} \cdot \mathrm{mL}^{-1}$ ultra pure Escherichia coli lipopolysaccharide (LPS) (Invivogen) for $18 \mathrm{~h}$ at $37^{\circ} \mathrm{C}$. LPS in this concentration did not induce significant cytokine release from the epithelial cell culture systems used (data not shown). For neutralisation assays, the supernatants were pre-treated with neutralising monoclonal antibodies (mAb) (anti-TNF- $\alpha$, anti-IL-1 $\beta$ or both) or mouse immunoglobulin (Ig)G (all R\&D Systems, Wiesbaden-Nordenstadt, Germany) for $60 \mathrm{~min}$. Pseudomonas aeruginosa PAO1 was grown to an optial density of $600 \mathrm{~mm}$ (OD600) of 0.8 in Luria-Bertani (LB) medium. Streptococcus pneumoniae PN36 was grown to an OD600 of 0.3 in soy broth supplemented with $10 \%$ FCS. For some experiments bacteria were heat-inactivated for $30 \mathrm{~min}$ at $96^{\circ} \mathrm{C}$. Cells were stimulated with the TLR-ligands (Invivogen, San Diego, CA, USA) flagellin $\left(100 \mathrm{ng} \cdot \mathrm{mL}^{-1}\right)$, peptidoglycan (TLR2 ligand, $\left.1 \mu \mathrm{g} \cdot \mathrm{mL}^{-1}\right)$ or FSL-1 $\left(200 \mathrm{ng} \cdot \mathrm{mL}^{-1}\right)$ for $6 \mathrm{~h}$. To test the effect of macrophage-derived supernatant on the epithelial cells, $1.5 \times 10^{5}$ cells (A549 or pAECs) were seeded per well of a 12well plate and incubated with the macrophage-derived supernatants for $18 \mathrm{~h}$. After washes and replacement with fresh medium (not conditioned), heat-inactivated bacteria ( $P$. aeruginosa PAO1 or S. pneumoniae PN36), flagellin, or peptidoglycan were applied for $6 \mathrm{~h}$. For co-culture experiments, $1 \times 10^{6}$ epithelial cells (A549 or pAECs) were seeded into each well of a six-well plate. $24 \mathrm{~h}$ after plating, $1 \times 10^{5}$ macrophages (U937 or monocyte-derived macrophages) were seeded on the epithelial cells. $6 \mathrm{~h}$ after seeding, attachment of the macrophages to the epithelial cell layer was confirmed by microscopy. Co-cultures were stimulated with heat-inactivated bacteria (P. aeruginosa PAO1 or S. pneumoniae PN36) for $24 \mathrm{~h}$.

\section{Fluorescence-activated cell sorter analysis}

pAECs were detached with PBS containing $3.7 \%$ EDTA at $37^{\circ} \mathrm{C}$. The cells were incubated with phycoerthrin-conjugated mouse mABs against TLR2 and TLR5 or with their corresponding isotype control (all eBioscience, San Diego, CA, USA) for $30 \mathrm{~min}$ at room temperature. To block unspecific binding of the antibodies, the samples were pre-incubated with $20 \mu \mathrm{g} \cdot \mathrm{mL}^{-1}$ purified mouse IgG (Dako, Hamburg, Germany). The cells were then washed twice with PBS containing 1\% FCS and $0.1 \% \mathrm{NaN}_{3}$, resuspended in CellFix (BD Pharmingen, Heidelburg, Germany) and analysed using a fluorescenceactivated cell sorter (FACSort flow cytometer; Becton Dickinson, Heidelberg, Germany).

\section{Bacterial survival assay}

Bacterial viability assays were used to determine the effect of macrophage-derived supernatants on the antimicrobial activity of airway epithelium. All experiments were performed using culture medium without antibiotics. After pre-incubation, cells were infected with living $P$. aeruginosa PAO1 or $S$. pneumoniae PN36, $40 \mu \mathrm{L}$ (corresponding to $5 \times 10^{4}$ colony forming units; CFU) were applied to the apical surface of the differentiated epithelium followed by incubation at $37^{\circ} \mathrm{C}$ for $6-8 \mathrm{~h}$. The cultures were then washed three times with $100 \mu \mathrm{L}$ of PBS and serial dilutions were plated onto LB agar plates. The plates were incubated overnight at $37^{\circ} \mathrm{C}$ and the numbers of $\mathrm{CFU}$ were counted.

\section{Determination of cytokine concentrations}

The concentrations of human IL- 6 , IL- 8 , TNF- $\alpha$ and IL- $1 \beta$ in the cell culture supernatants, as well as the levels of murine IL-6, keratinocyte chemoattractant (KC), TNF- $\alpha$ and IL-1 $\beta$ in culture supernatants and cell-free BALF, were determined by commercially available sandwich-type ELISA (R\&D Systems). 


\section{Real time RT-PCR}

Total RNA was purified using the RNeasy Mini Kit (Qiagen, Hilden, Germany) including treatment with RNase-free DNase set (Qiagen) and $1.5 \mu \mathrm{g}$ of total RNA was reverse transcribed using a cDNA synthesis kit (MBI Fermentas, St. Leon-Rot, Germany) applying oligo(dT)18. cDNA was diluted 1:5 and $5 \mu \mathrm{L}$ was used as template in a $25-\mu \mathrm{L}$ SYBR-Green-PCR mix, according to the manufacturer's instructions (ABgene, Hamburg, Germany). The following PCR primers were used: $\beta$-actin, 5'-AGCCTCGCCTTTGCCGA-3'; and 5'-CTGGTGCCTGGGGCG-3'; human $\beta$-defensin (hBD)-2, 5'-TCAGCTCCTGGTGAAGCTC-3' and 5'-GGGCAAAAGACTGGATGACA-3'; hBD-3, 5'-TATTATTGCAGAGTCAGAGGCG-3' and 5'-CACTCTCGTCATGTTTCAGGG-3'; TLR2, 5'-GAGAGTGGGAAATATGGACACCTT-3' and 5'-GCAGTTCCAAACATTCCACG-3'; TLR5, 5'-GAAAACCGCATTGCCAATAT-3' and 5'-TGCTGCAAATCGAGAATTT-3' (TIB Molbiol, Berlin, Germany). Specificity of RT-PCR was controlled by "no template" and "no reverse transcription" controls and melting curve analysis. Quantitative PCR results were obtained using the $\Delta \Delta C T$ method. Data were normalised to $\beta$-actin levels in each sample.

\section{Animals and infection models}

Animals were housed in a pathogen-free facility with single ventilated cages and received an endotoxin-free diet. All animal experiments were approved by the local federal authorities (Regierungspräsidium Giessen, Giessen, Germnay). To generate a constitutive knockout of RelA/p65 in myeloid cells, rela ${ }^{F / F}$ mice [21] were crossed with LysMCre animals [22] to generate a rela ${ }^{F / F}$ LysMCre mouse line. For the experiments sex-matched littermates from rela ${ }^{w t / F}$ LysMCre parents were used, the control animals were littermates with rela ${ }^{F / F}$ LysMCre or rela ${ }^{w t / w t}$ LysMCre. Mice were anesthetised and infected intranasally with viable or heat-inactivated $P$. aeruginosa PAO1 $\left(5 \times 10^{6} \mathrm{CFU}\right)$ in PBS or PBS alone as control. $24 \mathrm{~h}$ (for living bacteria) or $6 \mathrm{~h}$ (for heat-inactivated bacteria) after bacterial infection, the animals were euthanised, the tracheae were cannulated and a bronchoalveolar lavage (BAL) was performed (five times with $1 \mathrm{~mL}$ of PBS). Cytopreparations and immunohistochemistry were generated as described previously [20]. To determine the bacterial load of the lungs $24 \mathrm{~h}$ after infection, whole lungs were homogenised in $1 \mathrm{~mL}$ of PBS, serial dilutions were plated onto LB agar, and colonies were counted after incubation overnight. Murine alveolar macrophages were isolated by BAL and stimulated by LPS. Supernatants were applied to primary murine AECs. After washes, the cells were stimulated with flagellin and the release of $\mathrm{KC}$ was measured. AECs were isolated from resected tracheas [23]. Two tracheas were treated with Ham's F-12 (pen/strep, nystatin) containing $1.5 \mathrm{mg} \cdot \mathrm{mL}^{-1}$ pronase E (Sigma Aldrich) for $18 \mathrm{~h}$ at $4{ }^{\circ} \mathrm{C}$ and fetal bovine serum was added to a final concentration of $10 \%$. Cells from each of the six tracheas were pooled and collected by centrifugation at $400 \times g$ for $5 \mathrm{~min}$. Incubation was performed in petri dishes for $2 \mathrm{~h}$ to adhere ballast cells. The supernatant was collected and centrifuged at $400 \times g$ for $5 \mathrm{~min}$. Primary tracheal epithelial cells were resuspended in culture medium.

\section{p65 binding activity ELISA}

The DNA binding activity of the NF- $\mathrm{B}$ p65 subunit was measured by using a commercially available p65 ELISA
(TransAM p65 Transcription Factor ELISA; Active Motif, Rixensart, Belgium). Whole cell extracts from murine alveolar macrophages were prepared using a nuclear extract kit from Active Motif and normalised by protein concentration. The activity of p65 is expressed as absorbance at $450 \mathrm{~nm}$.

\section{Statistical analysis}

Data are presented as mean $\pm \mathrm{SD}$. Comparisons between groups were analysed by two-sided paired t-test or ANOVA for experiments with more than two subgroups. Post hoc range tests were performed with the two-sided paired t-test with Bonferroni adjustment. Results were considered statistically significant for $\mathrm{p}<0.05$.

\section{RESULTS}

Macrophages and epithelial cells are known to contribute to innate immunity of the lung. $\mathrm{NF}-\kappa \mathrm{B}$ is the prototypic transcription factor of immunity and regulates the expression of macrophage host defense responses [24]. To test whether macrophage activation is necessary for host defense and for epithelial activation in vivo, we evaluated the effect of selected truncation of the rela gene in myeloid cells in a murine pneumonia model. To characterise the course of the infection, we determined the numbers of viable bacteria and cells in the lavage. As a further outcome we determined the production of $\mathrm{KC}$ in epithelial cells. Infection of mice with inactivated myeloid NF- $\mathrm{KB}$ resulted in a significant increased number of viable bacterial in the lung homogenates compared with the wild-type (WT) mice (fig. 1a) with suppressed influx of inflammatory cells (fig. 1b). The relative number of individual cell types (macrophages, neutrophils and lymphocytes) was not statistically different between the two groups (data not shown). After inoculation of heat inactivated bacteria, the release of pro-inflammatory cytokines was largely diminished in the animals with suppressed macrophage function, TNF- $\alpha$ was virtually absent in the investigational group (fig. 1c). In contrast, in mice that were injected with viable bacteria, the higher load of bacterial in rela deficient mice resulted in significantly increased levels of cytokines (KO versus WT: IL-6, $343 \pm 152$ versus $31 \pm 23 \mathrm{pg} \cdot \mathrm{mL}^{-1} ;$ TNF- $\alpha, 46 \pm 15$ versus $\left.13 \pm 9 \mathrm{pg} \cdot \mathrm{mL}^{-1}\right)$. To determine the level of epithelial activation, we used immunohistochemical staining to detect KC in AECs. Mice with deleted myeloid rela showed significantly decreased signals of KC immunostaining in the epithelial cells (fig. 1d) compared with control animals (fig. $1 \mathrm{~d}$ and $1 \mathrm{e}$ ) indicating that the activity of macrophages is necessary to activate epithelial responses to microorganisms. To show that macrophages of mice with deleted myeloid rela are less responsive to bacterial stimulation, alveolar macrophages were isolated by lavage and exposed to LPS. The DNA binding activity of p65 was decreased in rela deficient cells (fig. 1f). These data show that myeloid RelA is important for host defense during a murine model of pneumonia. Macrophage activation also regulates the response of epithelial cells

\section{Macrophages activate AECs to recognise TLR ligands}

AECs form a barrier to the outside world and are continuously exposed to various microorganisms. Under stable conditions, the host defense mechanism provides a basic protection. AECs are intrinsically hyposensitive to pathogen-associated molecular pattern (PAMPs) and barley respond to the exposure to 

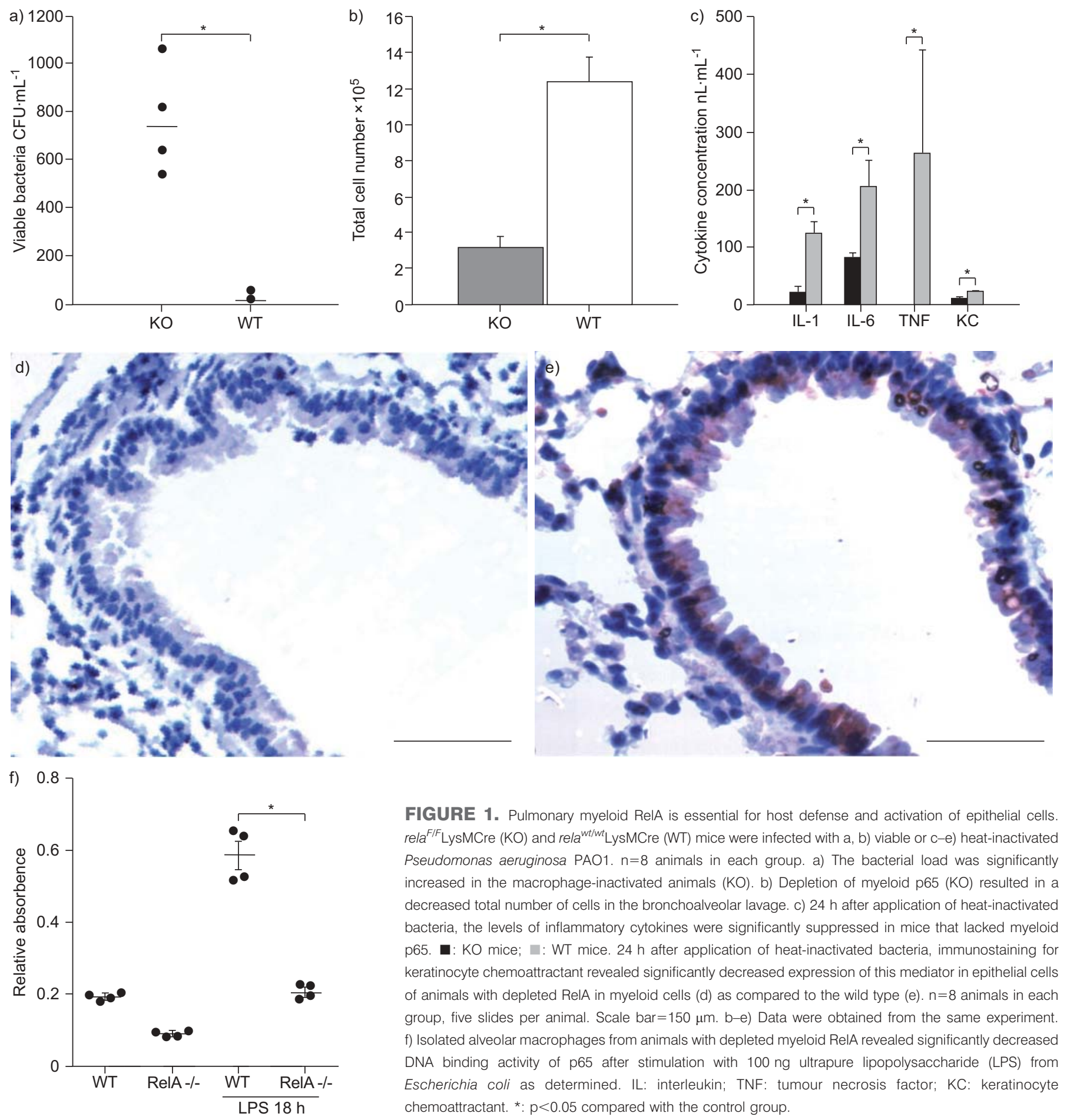

FIGURE 1. Pulmonary myeloid RelA is essential for host defense and activation of epithelial cells. rela ${ }^{F / F}$ LysMCre (KO) and rela ${ }^{\text {wt/wt }}$ LysMCre (WT) mice were infected with a, b) viable or c-e) heat-inactivated Pseudomonas aeruginosa PAO1. $\mathrm{n}=8$ animals in each group. a) The bacterial load was significantly increased in the macrophage-inactivated animals (KO). b) Depletion of myeloid p65 (KO) resulted in a decreased total number of cells in the bronchoalveolar lavage. c) $24 \mathrm{~h}$ after application of heat-inactivated bacteria, the levels of inflammatory cytokines were significantly suppressed in mice that lacked myeloid p65. - : KO mice; 1 : WT mice. $24 \mathrm{~h}$ after application of heat-inactivated bacteria, immunostaining for keratinocyte chemoattractant revealed significantly decreased expression of this mediator in epithelial cells of animals with depleted RelA in myeloid cells (d) as compared to the wild type (e). $n=8$ animals in each group, five slides per animal. Scale bar $=150 \mu \mathrm{m}$. b-e) Data were obtained from the same experiment. f) Isolated alveolar macrophages from animals with depleted myeloid RelA revealed significantly decreased DNA binding activity of p65 after stimulation with $100 \mathrm{ng}$ ultrapure lipopolysaccharide (LPS) from Escherichia coli as determined. IL: interleukin; TNF: tumour necrosis factor; KC: keratinocyte chemoattractant. *: $p<0.05$ compared with the control group.

microbes or their structures. These data have been obtained by several laboratories $[8,25]$ and could be replicated in our models. Nondifferentiated and ALI-culture differentiated AECs expressed the message of TLR 1-6, 9, 10 (fig. 2a). We then tested whether AECs respond to prototypical PAMPs that cause a dramatic effect when applied to macrophages. Peptidoglycan and flagellin, the ligands for TLR2 and TLR5, respectively, were applied and induced almost no induction of cytokine release by AECs (fig. 2b). Differentiated pAECs in ALI culture showed increased secretion of cytokines compared with cell grown pAECs submersed in medium (fig. 2b). In contrast, macrophages release abundant IL- 8 when stimulated with these molecular patterns (fig. 2b).

The close physical contact between macrophages and epithelial cells in the respiratory tract implies a functional interaction 
between both cell types. We applied various models to study the reciprocal interaction between these two cell types. A conventional co-culture model of A549 lung epithelial cells and U937 derived macrophages in submersed conditions resulted in a significantly increased release of IL-6 from the epithelial cell line A549 after exposure to Gram-positive or Gramnegative heat-inactivated bacteria (fig. 3a). This effect could also be seen in co-cultures using primary monocyte-derived macrophages and pAECs (fig. 3b). The effect of the co-culture was synergistic in comparison to the effect of bacterial application to the individual cells. These data indicate that epithelial cells and professional myeloid host defense cells cooperate during host defense.
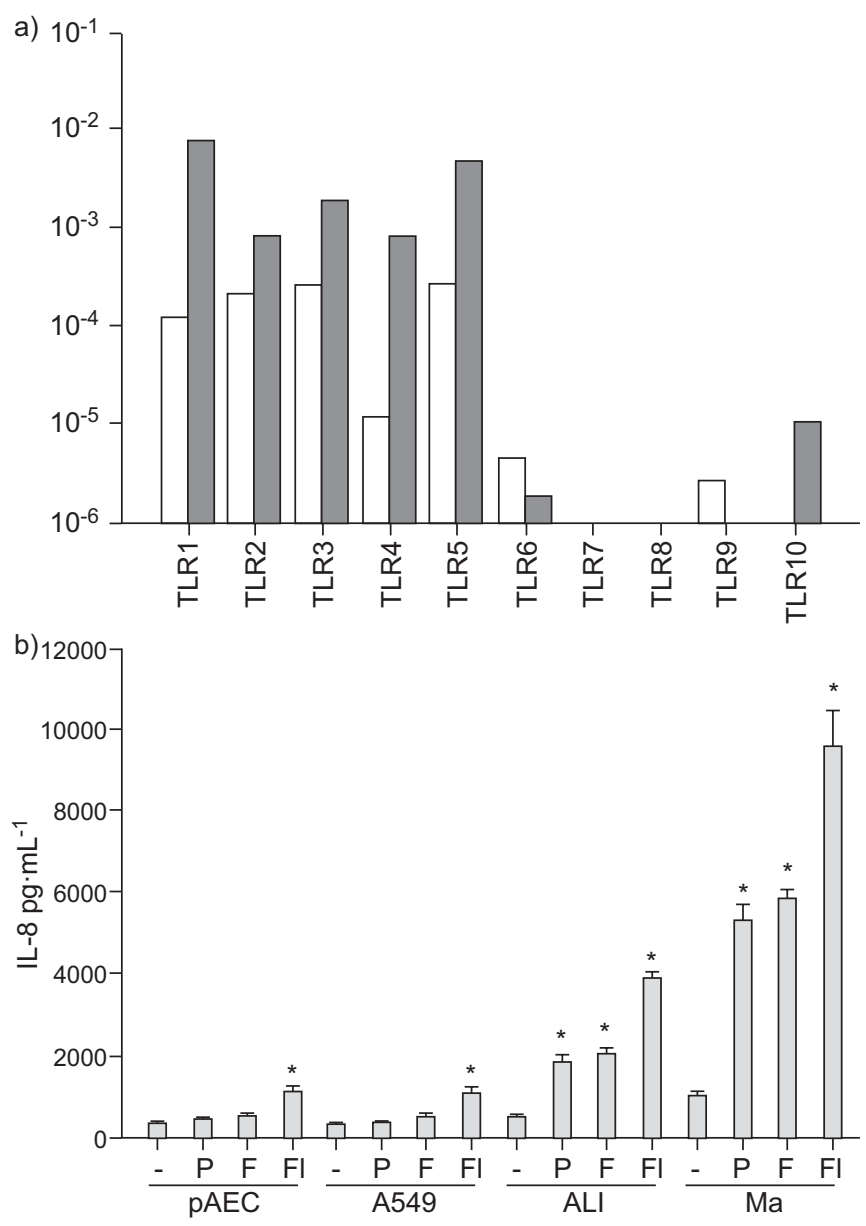

FIGURE 2. Airway epithelial cells (AECs) were hyporesponsive to microbial structures. a) Primary AEC expression of toll-like receptor (TLR) 1-10 as determined by RT-PCR. Primary AECs in submersed nondifferentiated culture $(\square)$ and differentiated primary AECs in air liquid interface culture ( $\square$ ) showed the presence of transcripts of human TLR 1-6, 9, and 10 (representative results out of five experiments). b) Primary AECs in submersed culture (PAEC), differentiated primary AECs in air liquid interface culture (ALI) and the cell line A549 responded poorly to exposure to peptidoglycan (P), FSL-1 (F) or flagellin (FI), whereas macrophages (Ma) secreted abundant interleukin (IL)-8. $n=10$ animals in each group. *: $p<0.05$ compared with the control group (-).

\section{Soluble factors from macrophages induce TLR signalling in AECs}

AECs can be activated by macrophages to recognise PAMPs and respond to the release of host defense molecules and proinflammatory mediators. To test whether soluble factors in the medium are sufficient to activate AECs, macrophage-conditioned medium (mcM) was applied. The response of AECs to PAMPs as measured by the release of IL- 8 and IL- 6 was significantly stimulated by the pre-incubation with $\mathrm{mcM}$ from macrophages stimulated by LPS, whereas mcM from unstimulated "resting" macrophages had only little or no effect (data not shown) (fig. 4a and b). Of note, the epithelial response without mcM was virtually absent. To determine whether the same mechanisms regulate the expression of epithelial host defense molecules, we determined the transcript levels of the mucosal defensins hBD-2 and hBD-3 in epithelial cells. The application of mcM sensitised the AECs for the production of hBD-2 and hBD-3 (fig. 4c). To test whether alveolar macrophages from rela deficient animals mice fail to activate AECs, macrophages were isolated by lavage and stimulated with LPS. The conditioned medium together with the TLR ligand flagellin was used to stimulate primary murine epithelial cells and the secretion of KC was measured. We found that alveolar macrophages from rela deficient animals are significantly less capable of stimulating epithelial cells (fig. 4g).

It is clearly established that detection of PAMPs used in the present study was mediated by TLRs [26]. The induction of gene transcription and the regulation of the protein's surface expression are possible mechanisms to increase the sensibility of a cell to TLR ligands. We tested whether increased availability of these receptors is linked to the activated state of AECs. While the exposure to flagellin and peptidoglycan alone did not change the mRNA levels of TLR2 or TLR5, the application of mcM together with the TLR ligand resulted in increased expression of the TLR message (fig. 4d). Exposure to $\mathrm{mcM}$, together with the respective ligand, also increased surface expression of TLR2 and TLR5 pAECs as determined by fluorescence-activated cell sorter analysis (fig. 4e and f).

Soluble factors secreted from macrophages activate epithelial cells to increase their sensitivity to detect PAMPs. TNF- $\alpha$ and IL$1 \beta$ are classical acute phase cytokines that are expressed by macrophages. We tested whether the effects of the mcM could be blocked by neutralising antibodies that inactivate these cytokines. The induced expression of TLR2 or TLR5 by mcM together with the respective ligand was significantly suppressed by the application of anti- TNF- $\alpha$, whereas anti-IL-1 $\beta$ had no effect (fig. 5a). The combination of anti-TNF- $\alpha$ and anti-IL-1 $\beta$ showed the same effect as anti-TNF- $\alpha$ alone. The exogenous application of TNF- $\alpha$ together with the ligands induced increased expression of the TLRs (fig. 5a). To test whether the principle mechanism is also relevant in differentiated cells, we exposed AECs in ALI culture with TNF- $\alpha$ and determined the expression of TLR2 or TLR5 after exposure to the respective TLR ligands. Preincubation with TNF- $\alpha$ significantly augmented the expression of TLR2 and TLR5 (fig. 5b).

\section{Macrophages regulate epithelial host defense}

Epithelial cells secrete inflammatory mediators that regulate innate and adaptive host defense mechanisms. The induction of this activity is largely dependent on the regulation by 

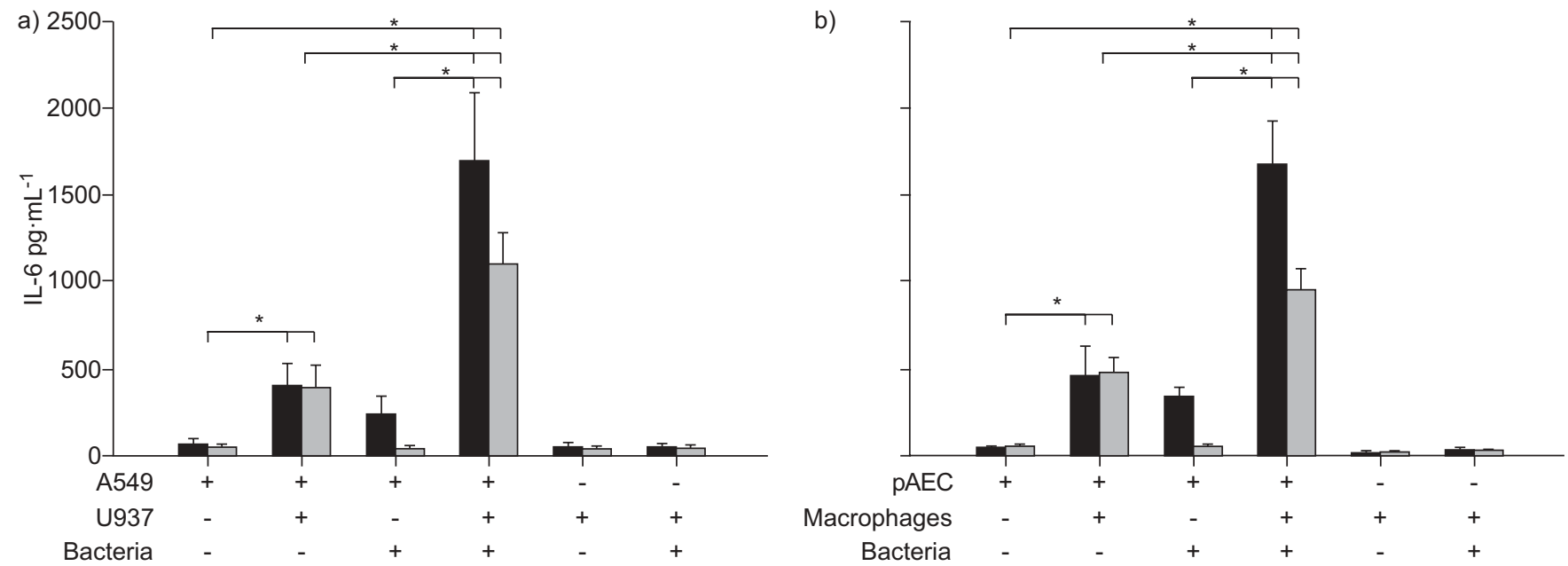

FIGURE 3. Macrophages activate airway epithelial cells (AECs) to respond to microbial structures. a) Submersed co-culture of the epithelial cell line A549 together with U937-derived macrophages resulted in significantly increased release of interleukin (IL)- 6 after the addition of heat inactivated bacteria. b) Stimulation of a submersed coculture of primary AECs (PAECs) and peripheral blood mononuclear cell-derived macrophages also resulted in a significant increase of IL-6 secretion. $\mathbf{\square}$ : Pseudomonas aeruginosa PAO1; ${ }^{0}$ : Staphylococcus pneumoniae PN36. $n=4$ animals in each group. *: $p<0.05$.

macrophages. Epithelial cells also provide effective host defense measures by the production of antimicrobial substances, such as antimicrobial peptides. To test whether the amount of epithelial host defense depends on macrophage factors, we infected differentiated airway epithelium with bacteria and determined the number of viable microorganisms after $6 \mathrm{~h}$. AECs in ALI culture exhibited significant antimicrobial activity [27]. We found that pre-incubation of AECs with $\mathrm{mcM}$ increased the host defense activity of the epithelium against $P$. aeruginosa (fig. $5 \mathrm{c}$ ). $\mathrm{mcM}$ from LPS-stimulated macrophages had a stronger effect, LPS applied to AECs had no effect.

\section{DISCUSSION}

The main finding of our study was that myeloid RelA is required to elicit an effective host defense response during pneumonia and that macrophages regulate epithelial sensitivity to microbial patterns. Antimicrobial activity, cytokine levels and neutrophil influx were decreased in animals with defective myeloid RelA. Macrophages sensitise epithelial cells for the detection of bacterial components and subsequent host defense reactions. This effect is mediated by soluble factors released by macrophages and TNF- $\alpha$ appears to be a major mediator of this effect. We used animal models and further characterised mechanisms in human tissue, underscoring the relevance of the identified pathways in humans and animal models.

A number of studies have investigated the relative role of epithelial versus professional host defense cells in pulmonary host defense mechanisms and showed that both cell compartments contribute [9-16]. The present data agree with these observations in that myeloid cells (mainly macrophages and neutrophils) are critical effector cells during lung infection. RelA is part of the canonical NF- $\kappa B$ pathway that is the prototypical immune transcription pathway. RelA and p50 have been identified from nuclear extracts of lungs following bacterial exposure [28]. The present data highlight the role of macrophages as immune sentinels that activate epithelial cells.

Epithelial cells are primarily considered as structural cells. While the formation of a physical barrier is probably a critical function of these cells, epithelial cells are capable of engaging in inflammatory and host defense reactions [10, 29]. Epithelial cells of the respiratory or the gastrointestinal tract are physiologically hyporesponsive to microbial patterns $[8,30$, 31]. This appears to be teleologically reasonable because a strong inflammatory reaction to the constant exposure of inhaled microbial components could cause organ destruction. In the case of microbial invasion and infection, epithelial cells can produce a significant amount of inflammatory mediators, mostly CXC chemokines [9], and produce antimicrobial substances [2]. Macrophages are capable of activating epithelial cells through the release of TNF- $\alpha$ and IL- $1 \beta$, which induce NF-kB [32] and the expression of the defensin hBD-2 [33]. The epithelial host defense reaction is complex and involves numerous factors. In models of acute lung injury [34] and LPS exposure [35], macrophage derived TNF- $\alpha$ was identified as the main mediator responsible for the activation of epithelial cells. In the present study, host defense reactions were significantly suppressed in animals with depleted myeloid RelA, probably as a result of the breach of antimicrobial activity of both macrophages/neutrophils and epithelial cells. IL-1 $\beta$ and TNF- $\alpha$ levels in the lavage were significantly decreased in the genetically modified animals exposed to heat-inactivated bacteria. The blunted induction of epithelial $\mathrm{KC}$ underscores the regulator effect that macrophages have on epithelial cells. Macrophage-derived cytokines are probably responsible for stimulation of epithelial cells. Isolated alveolar macrophages from these rela deficient animals stimulated AECs significantly less in comparison to cells from WT animals. These mechanisms were also identified in the culture model using human material. In our study, TNF- $\alpha$ appeared to have a more prominent role. Animal studies with depletion of 

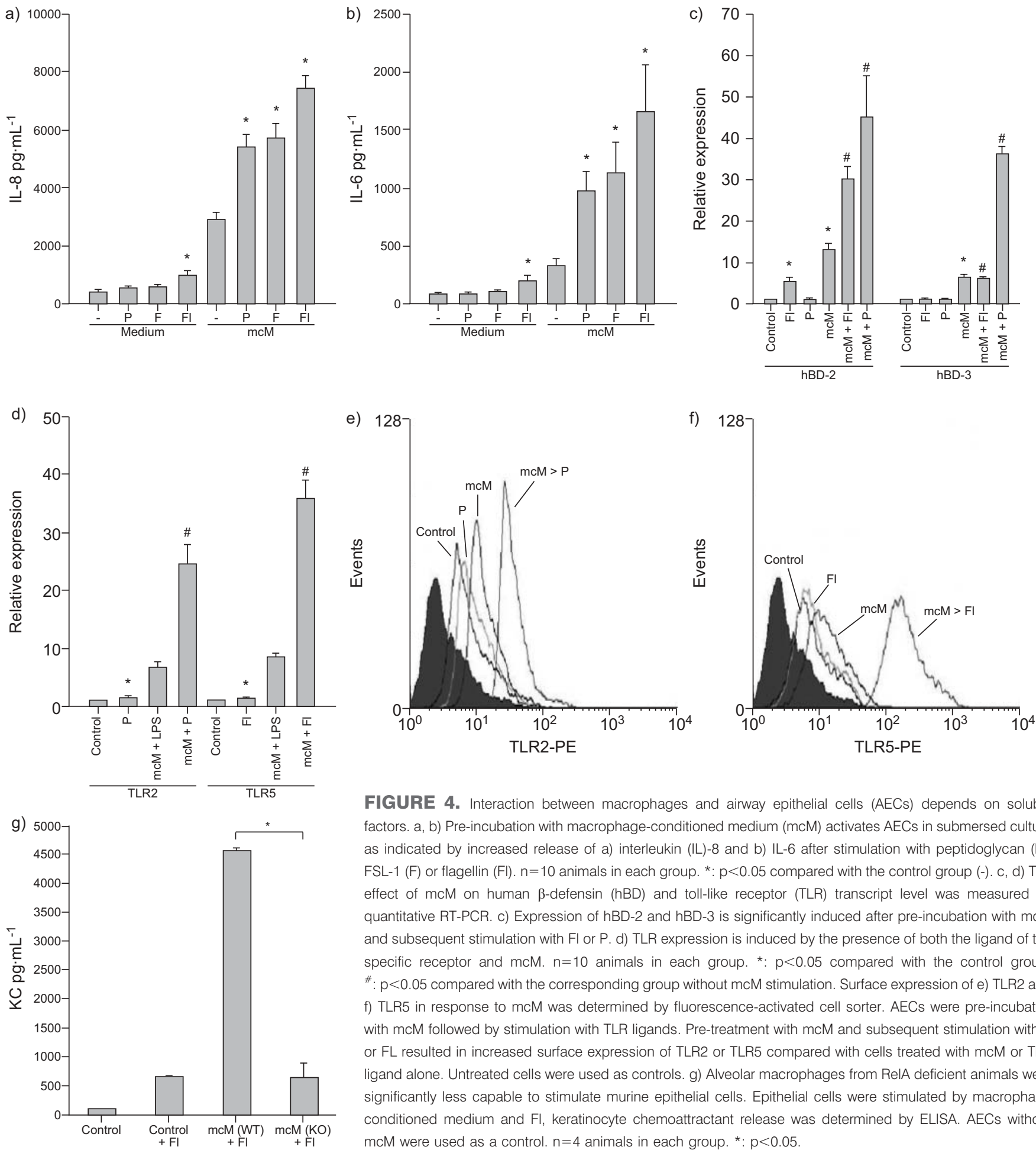

FIGURE 4. Interaction between macrophages and airway epithelial cells (AECs) depends on soluble factors. a, b) Pre-incubation with macrophage-conditioned medium ( $\mathrm{mcM}$ ) activates AECs in submersed culture as indicated by increased release of a) interleukin (IL)-8 and b) IL-6 after stimulation with peptidoglycan (P), FSL-1 (F) or flagellin (FI). $n=10$ animals in each group. *: $p<0.05$ compared with the control group (-). c, d) The effect of mcM on human $\beta$-defensin (hBD) and toll-like receptor (TLR) transcript level was measured by quantitative RT-PCR. c) Expression of hBD-2 and hBD-3 is significantly induced after pre-incubation with mcM and subsequent stimulation with $\mathrm{Fl}$ or P. d) TLR expression is induced by the presence of both the ligand of the specific receptor and mcM. $n=10$ animals in each group. *: $p<0.05$ compared with the control group; \#: $p<0.05$ compared with the corresponding group without mcM stimulation. Surface expression of e) TLR2 and f) TLR5 in response to mcM was determined by fluorescence-activated cell sorter. AECs were pre-incubated with $\mathrm{mcM}$ followed by stimulation with TLR ligands. Pre-treatment with $\mathrm{mcM}$ and subsequent stimulation with $\mathrm{P}$ or FL resulted in increased surface expression of TLR2 or TLR5 compared with cells treated with mcM or TLR ligand alone. Untreated cells were used as controls. g) Alveolar macrophages from RelA deficient animals were significantly less capable to stimulate murine epithelial cells. Epithelial cells were stimulated by macrophage conditioned medium and $\mathrm{Fl}$, keratinocyte chemoattractant release was determined by ELISA. AECs without mcM were used as a control. $n=4$ animals in each group. *: $p<0.05$.

macrophages showed that these cells significantly contribute to the production of IL-1 $\beta$ and TNF- $\alpha[36,37]$.

Microbial tolerance of epithelial cells is an intriguing phenomenon, whose mechanism is largely unclear. AECs are under constant exposure to inhaled microorganisms and innate host defense mechanisms result in the elimination of inhaled microorganisms without frank inflammation, which would compromise the function of the organ by continuous inflammation and tissue destruction. Our data show that the surface expression of TLR is one factor that regulates the sensitivity of epithelial cells to microbial compounds. Several other mechanisms have been described that can result in microbial tolerance of epithelial cells. MD2 is necessary for the recognition of TLR4 by epithelial cells and low expression levels might limit the TRL function [25]. Epithelia consist of polarised cells that 

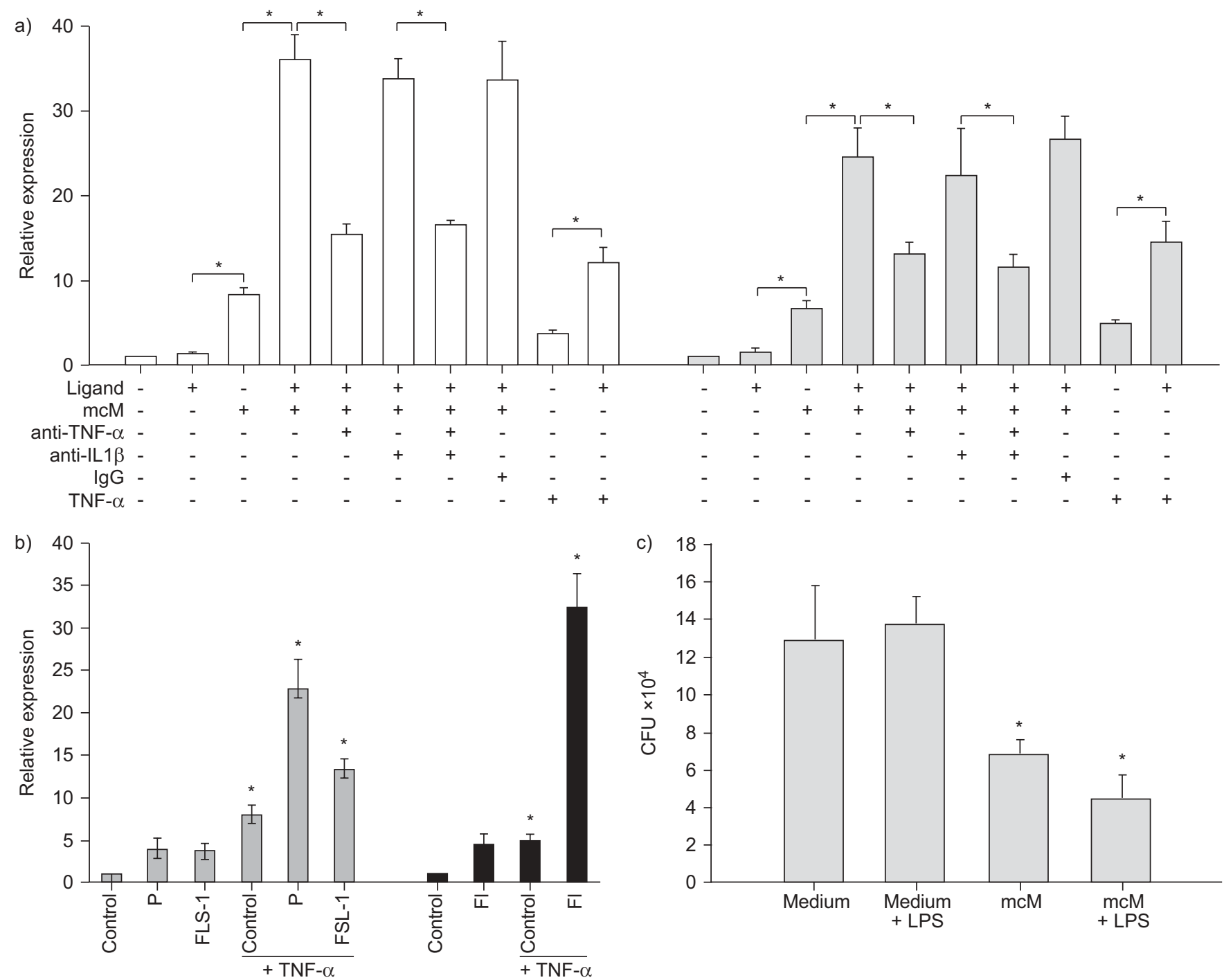

FIGURE 5. Effects of macrophages on primary airway epithelial cells (AECs) are mediated by tumour necrosis factor (TNF)- $\alpha$ and are functionally relevant. a) Expression of toll-like receptor (TLR)2 ( $\square$ ) or TLR5 $(\square)$ transcripts by macrophage-conditioned medium (mcM) together with the respective ligand is significantly suppressed by the application of anti-TNF- $\alpha$. External application of TNF- $\alpha$ induces expression of the TLRs. Anti-interleukin (IL)- $1 \beta$ or the control immunoglobulin (Ig)G had no additional effect. $n=10$ animals in each group. ${ }^{*}: p<0.05$ compared with the indicated control group. b) Differentiated primary AECs in air liquid interface were incubated with TNF- $\alpha$ and then stimulated by peptidoglycan (P), FSL-1 (F) or flagellin (FI). The expression of TLR-2 ( $\square$ ) or TLR-5 ( $\square$ ) was determined by quantitve RT-PCR. $n=10$ animals in each group. *: $p<0.05$ compared with the indicated control group without TNF- $\alpha$. c) Differentiated airway epithelium (primary AECs in air-liquid interface culture) was used to determine the functional effect of the interaction between macrophages and AECs. The cells were infected with Pseudomonas aeruginosa and the numbers of viable bacteria were determined after $6 \mathrm{~h}$. Co-incubation with mcM induced the antimicrobial activity. $\mathrm{n}=4$ animals in each group. ${ }^{*}: \mathrm{p}<0.05$ compared with the control group.

separate distinct compartments. Interaction with microorganisms from the apical (luminal, outside) surface results in different cellular consequences in comparison to the contact at the basolateral side [38]. Our data suggest that regulation on the transcriptional levels is responsible for the amount of receptor present at the cell surface. The lack of apical surface expression of pattern recognition receptors can also be due to intracellular location of TLRs, TLR2 is localised to intracellular compartments and enriched in caveolin-1-associated lipid raft microdomains presented on the apical surface of AECs after bacterial infection [5], or segregation of receptors to the basolateral membrane compartment [38]. A number of molecules are also known to interact with the TLR signalling, including SIGIRR [39], TRIAD3 or RP105 [40]. In addition, intracellular proteins such as IRAKM, PI(3)K, Tollip, SOCS1 and sMyD88 downregulate TLR signalling [41]. As shown in our study, soluble macrophage mediators increase the level of TLR transcript, protein, and surface expression. This increased availability of TLR results in epithelial engagement in inflammation and host defense reactions.

In conclusion, mucosal barrier and host defense functions are regulated within a network composed of different cell types [42] Under steady-state-conditions, epithelial cells create a physical barrier and are relatively tolerant to the presence of luminal microbes. Macrophage activation through the canonical NF- $\kappa B$ 
pathways is critical for an effective immune reaction during pneumonia. In addition to their direct antimicrobial activities, myeloid cells liberate epithelial innate host defense and inflammatory activities. This activation is mediated by soluble mediators and includes increased expression of TLRs and sensitive recognition of microbial patterns with subsequent release of inflammatory mediators and defensin. This network concept allows a more detailed insight into mucosal immunity to be developed. Disruption of the homeostatic balance between barrier and inflammation probably contributes to the development of lung diseases that are often characterised by a broken epithelial barrier and uncontrolled inflammation. Examples for this situation are common diseases, such as asthma, chronic obstructive lung disease or lung cancer.

\section{SUPPORT STATEMENT}

This study was supported by grants from the Deutsche Forschungsgemeinschaft (Ba 1641/8-1; SFB/IR 22) and the German Federal Ministry of Education and Research (Bundesministerium für Bildung und Forschung; both Bonn, Germany) through the German research network PROGRESS, which was awarded to R. Bals.

\section{STATEMENT OF INTEREST}

None declared.

\section{ACKNOWLEDGEMENTS}

We would like to thank J. Behr (University of Munich, Munich, Germany) for providing primary epithelial cells and T. Damm (University of Marburg, Marburg, Germany) for excellent technical support.

\section{REFERENCES}

1 Medzhitov R, Janeway C Jr. Innate immunity. N Engl J Med 2000; 343: 338-344.

2 Bals R, Weiner DJ, Wilson JM. The innate immune system in cystic fibrosis lung disease. J Clin Invest 1999; 103: 303-307.

3 Lambrecht BN. Alveolar macrophage in the driver's seat. Immunity 2006; 24: 366-368.

4 Greene CM, McElvaney NG. Toll-like receptor expression and function in airway epithelial cells. Arch Immunol Ther Exp (Warsz) 2005; 53: 418-427.

5 Soong G, Reddy B, Sokol S, et al. TLR2 is mobilized into an apical lipid raft receptor complex to signal infection in airway epithelial cells. J Clin Invest 2004; 113: 1482-1489.

6 Platz J, Beisswenger C, Dalpke A, et al. Microbial DNA induces a host defense reaction of human respiratory epithelial cells. J Immunol 2004; 173: 1219-1223.

7 Opitz B, Puschel A, Schmeck B, et al. Nucleotide-binding oligomerization domain proteins are innate immune receptors for internalized Streptococcus pneumoniae. J Biol Chem 2004; 279: 36426-36432.

8 Mayer AK, Muehmer M, Mages J, et al. Differential recognition of TLR-dependent microbial ligands in human bronchial epithelial cells. J Immunol 2007; 178: 3134-3142.

9 Hajjar AM, Harowicz H, Liggitt HD, et al. An essential role for non-bone-marrow-derived cells in control of pseudomonas aeruginosa pneumonia. Am J Respir Cell Mol Biol 2005; 33: 470-475.

10 Noulin N, Quesniaux VF, Schnyder-Candrian S, et al. Both hemopoietic and resident cells are required for MyD88-dependent pulmonary inflammatory response to inhaled endotoxin. $J$ Immunol 2005; 175: 6861-6869.

11 Hollingsworth JW, Chen BJ, Brass DM, et al. the critical role of hematopoietic cells in lipopolysaccharide-induced airway inflammation. Am J Respir Crit Care Med 2005; 171: 806-813.
12 Poynter ME, Irvin CG, Janssen-Heininger YM. A prominent role for airway epithelial NF-kappa B activation in lipopolysaccharideinduced airway inflammation. J Immunol 2003; 170: 6257-6265.

13 Alcamo E, Mizgerd JP, Horwitz BH, et al. Targeted mutation of TNF receptor I rescues the RelA-deficient mouse and reveals a critical role for NF-kappa B in leukocyte recruitment. J Immunol 2001; 167: 1592-1600.

14 Skerrett SJ, Liggitt HD, Hajjar AM, et al. Respiratory epithelial cells regulate lung inflammation in response to inhaled endotoxin. Am J Physiol Lung Cell Mol Physiol 2004; 287: L143-L152.

15 Sadikot RT, Han W, Everhart MB, et al. Selective I kappa B kinase expression in airway epithelium generates neutrophilic lung inflammation. J Immunol 2003; 170: 1091-1098.

16 Sadikot RT, Zeng H, Joo M, et al. Targeted immunomodulation of the NF-kappaB pathway in airway epithelium impacts host defense against Pseudomonas aeruginosa. J Immunol 2006; 176: 4923-4930.

17 Nys M, Deby-Dupont G, Habraken Y, et al. Bronchoalveolar lavage fluids of patients with lung injury activate the transcription factor nuclear factor-kappaB in an alveolar cell line. Clin Sci (Lond) 2002; 103: 577-585.

18 Knapp S, Wieland CW, van't Veer C, et al. Toll-like receptor 2 plays a role in the early inflammatory response to murine pneumococcal pneumonia but does not contribute to antibacterial defense. J Immunol 2004; 172: 3132-3138.

19 Quinton LJ, Jones MR, Simms BT, et al. Functions and regulation of NF-kappaB RelA during pneumococcal pneumonia. J Immunol 2007; 178: 1896-1903.

20 Beisswenger C, Kandler $\mathrm{K}$, Hess C, et al. Allergic airway inflammation inhibits pulmonary antibacterial host defense. $J$ Immunol 2006; 177: 1833-1837.

21 Algul H, Treiber M, Lesina M, et al. Pancreas-specific RelA/p65 truncation increases susceptibility of acini to inflammationassociated cell death following cerulein pancreatitis. J Clin Invest 2007; 117: 1490-1501.

22 Clausen BE, Burkhardt $\mathrm{C}$, Reith $\mathrm{W}$, et al. Conditional gene targeting in macrophages and granulocytes using LysMcre mice. Transgenic Res 1999; 8: 265-277.

23 You Y, Richer EJ, Huang T, et al. Growth and differentiation of mouse tracheal epithelial cells: selection of a proliferative population. Am J Physiol Lung Cell Mol Physiol 2002; 283: L1315-L1321.

24 Christman JW, Sadikot RT, Blackwell TS. The role of nuclear factor-kappa B in pulmonary diseases. Chest 2000; 117: 1482-1487.

25 Jia HP, Kline JN, Penisten A, et al. Endotoxin responsiveness of human airway epithelia is limited by low expression of MD-2. Am J Physiol Lung Cell Mol Physiol 2004; 287: L428-L437.

26 Akira S, Uematsu S, Takeuchi O. Pathogen recognition and innate immunity. Cell 2006; 124: 783-801.

27 Bals R, Weiner DJ, Meegalla RL, et al. Transfer of a cathelicidin peptide antibiotic gene restores bacterial killing in a cystic fibrosis xenograft model. J Clin Invest 1999; 103: 1113-1117.

28 Blackwell TS, Lancaster LH, Blackwell TR, et al. Differential NFkappaB activation after intratracheal endotoxin. Am J Physiol 1999; 277: L823-L830.

29 Sadikot RT, Han W, Everhart MB, et al. Selective I kappa B kinase expression in airway epithelium generates neutrophilic lung inflammation. J Immunol 2003; 170: 1091-1098.

30 Rescigno M, Urbano M, Valzasina B, et al. Dendritic cells express tight junction proteins and penetrate gut epithelial monolayers to sample bacteria. Nat Immunol 2001; 2: 361-367.

31 Otte JM, Cario E, Podolsky DK. Mechanisms of cross hyporesponsiveness to Toll-like receptor bacterial ligands in intestinal epithelial cells. Gastroenterology 2004; 126: 1054-1070.

32 Ishii H, Fujii T, Hogg JC, et al. Contribution of IL-1 beta and TNFalpha to the initiation of the peripheral lung response to atmospheric particulates (PM10). Am J Physiol Lung Cell Mol Physiol 2004; 287: L176-L183. 
33 Tsutsumi-Ishii Y, Nagaoka I. Modulation of human beta-defensin-2 transcription in pulmonary epithelial cells by lipopolysaccharidestimulated mononuclear phagocytes via proinflammatory cytokine production. J Immunol 2003; 170: 4226-4236.

34 Sharma AK, Fernandez LG, Awad AS, et al. Proinflammatory response of alveolar epithelial cells is enhanced by alveolar macrophage-produced TNF-alpha during pulmonary ischemiareperfusion injury. Am J Physiol Lung Cell Mol Physiol 2007; 293: L105-L113.

35 Elizur A, Adir-Kirk TL, Kelley DG, et al. Tumour necrosis factoralpha from macrophages enhances lps-induced clara cell expression of keratinocyte-derived chemokine. Am J Respir Cell Mol Biol 2008; 38: 8-15.

36 Hashimoto S, Pittet JF, Hong K, et al. Depletion of alveolar macrophages decreases neutrophil chemotaxis to Pseudomonas airspace infections. Am J Physiol 1996; 270: L819-L828.
37 Lentsch AB, Czermak BJ, Bless NM, et al. Essential role of alveolar macrophages in intrapulmonary activation of NF-kappaB. Am J Respir Cell Mol Biol 1999; 20: 692-698.

38 Lee J, Mo JH, Katakura K, et al. Maintenance of colonic homeostasis by distinctive apical TLR9 signalling in intestinal epithelial cells. Nat Cell Biol 2006; 8: 1327-1336.

39 Xiao H, Gulen MF, Qin J, et al. The toll-interleukin-1 receptor member SIGIRR regulates colonic epithelial homeostasis, inflammation, and tumorigenesis. Immunity 2007; 26: 461-475.

40 Divanovic S, Trompette A, Atabani SF, et al. Inhibition of TLR-4/ MD-2 signaling by RP105/MD-1. J Endotoxin Res 2005; 11: 363-368.

41 Han J, Ulevitch RJ. Limiting inflammatory responses during activation of innate immunity. Nat Immunol 2005; 6: 1198-1205.

42 Shaykhiev R, Bals R. Interactions between epithelial cells and leukocytes in immunity and tissue homeostasis. J Leukoc Biol 2007; 82: 1-12. 